\title{
Holmium Ho 166 Poly(L-Lactic Acid) Microspheres
}

National Cancer Institute

\section{Source}

National Cancer Institute. Holmium Ho 166 Poly(L-Lactic Acid) Microspheres. NCI

Thesaurus. Code C88336.

Holmium Ho166 containing poly l-lactic acid (PLA) microspheres with potential antineoplastic actvity. Upon intra-arterial hepatic administration of holmium 166 microspheres, this agent is able to emit both beta particles direct killing cells and gamma photons for nuclear imaging. In addition, since holmium 166 is paramagnetic, this agent can be used for magnetic resonance imaging (MRI). 